\title{
Testing the inverted neutrino mass ordering with neutrinoless double- $\beta$ decay
}

\author{
Matteo Agostini $\odot,{ }^{1, *}$ Giovanni Benato $\odot,{ }^{2, \dagger}$ Jason A. Detwiler $\odot,{ }^{3, \dagger}$ Javier Menéndez $\odot,{ }^{4, \S}$ and Francesco Vissani $\odot^{2,5, \|}$ \\ ${ }^{1}$ Department of Physics and Astronomy, University College London, Gower Street, London WC1E 6BT, United Kingdom \\ ${ }^{2}$ INFN, Laboratori Nazionali del Gran Sasso, 67100 Assergi, L'Aquila, Italy \\ ${ }^{3}$ Center for Experimental Nuclear Physics and Astrophysics and Department of Physics, \\ University of Washington, Seattle, Washington 98115, USA \\ ${ }^{4}$ Department of Quantum Physics and Astrophysics and Institute of Cosmos Sciences, University of Barcelona, 08028 Barcelona, Spain \\ ${ }^{5}$ Gran Sasso Science Institute, 67100 L'Aquila, Italy
}

(Received 7 July 2021; accepted 23 September 2021; published 11 October 2021)

\begin{abstract}
We quantify the extent to which future experiments will test the existence of neutrinoless double- $\beta$ decay mediated by light neutrinos with inverted-ordered masses. While it remains difficult to compare measurements performed with different isotopes, we find that future searches will fully test the inverted-ordering scenario, as a global, multi-isotope endeavor. They will also test other possible mechanisms driving the decay, including a large uncharted region of the allowed parameter space assuming that neutrino masses follow the normal ordering.
\end{abstract}

DOI: 10.1103/PhysRevC.104.L042501

Neutrino oscillation [1-4] proves that the neutrino has mass and the standard model of particle physics is incomplete. The unknown origin of the neutrino mass has drawn enormous attention to neutrinoless double- $\beta(0 v \beta \beta)$ decay, a matter-creating nuclear transition in which two neutrons decay simultaneously into two protons, emitting only two new electrons and no antineutrinos [5]. The discovery of $0 v \beta \beta$ decay would establish that the neutrino is its own antiparticle and has a Majorana mass [6]. It would also mark the first observation of a lepton-creating process, proving that neither lepton number $(L)$ nor baryon minus lepton number $(B-L)$ are symmetries of the standard model, as predicted by leading theories explaining the matter-antimatter asymmetry of our universe [7]. Indeed, searching for $0 \nu \beta \beta$ decay is the most sensitive experimental approach to test Majorana neutrino masses and their associated $L$ violation. It is also a unique probe of new physics at ultrahigh energy scales not accessible by current accelerators $[8,9]$.

Different physics mechanisms can lead to $0 v \beta \beta$ decay [10]. However, the exchange of light Majorana neutrinos interacting via standard, weak left-handed currents plays a special role. It is the only mechanism allowed by all theories

\footnotetext{
*matteo.agostini@ucl.ac.uk

† giovanni.benato@lngs.infn.it

¥jasondet@uw.edu

§menendez@fqa.ub.edu

"vissani@lngs.infn.it
}

Published by the American Physical Society under the terms of the Creative Commons Attribution 4.0 International license. Further distribution of this work must maintain attribution to the author(s) and the published article's title, journal citation, and DOI. Funded by $S C O A P^{3}$. predicting $0 \nu \beta \beta$ decay, and typically dominates the rate of the process $[11,12]$. Assuming that the decay is mediated by light neutrinos, its half-life is [13]

$$
T_{1 / 2}^{-1}=G g_{A}^{4} M^{2} \frac{m_{\beta \beta}^{2}}{m_{e}^{2}},
$$

where $G$ is the phase-space integral, $g_{A} \simeq 1.276$ [14] is the axial-vector coupling, $M$ is the nuclear matrix element, and $m_{\beta \beta}$ is the effective Majorana mass, normalized for convenience by the electron mass $m_{e}$. The Majorana mass captures the physics of the exchanged neutrinos and is a function of the neutrino oscillation parameters, the neutrino mass eigenvalues $m_{i}$, and the Majorana phases: $m_{\beta \beta}=\left|\sum_{i} U_{e i}^{2} m_{i}\right|$, where $U_{e i}$ are the elements of the full 6-parameter PMNS matrix [15].

Neutrino oscillation measurements constrain the range of allowed $m_{\beta \beta}$ values [16] and prove that $\left|U_{e 3}^{2}\right| \ll\left|U_{e 2}^{2}\right|<\left|U_{e 1}^{2}\right|$ and $m_{2}^{2}-m_{1}^{2} \ll\left|m_{3}^{2}-m_{2}^{2}\right|$ [17-26]. This implies that the effective Majorana mass is strictly larger than zero if neutrino masses follow the inverted ordering, i.e., $m_{3}<m_{1} \lesssim m_{2}$. In this case, the lowest $m_{\beta \beta}$ value, minimized with respect to the unknown Majorana phases and $m_{3}$, is given by

$$
\left(m_{\beta \beta}^{\min }\right)_{\mathrm{IO}}=\left|U_{e 1}^{2}\right| m_{1}-\left|U_{e 2}^{2}\right| m_{2}-\left|U_{e 3}^{2}\right| m_{3},
$$

with $m_{3}=\left|U_{e 3}^{2}\right| /\left(\left|U_{e 1}^{2}\right| / m_{1}-\left|U_{e 2}^{2}\right| / m_{2}\right) \approx 3 \mathrm{meV}$. Using the latest values and uncertainties from the Particle Data Group [4], we obtain

$$
\left(m_{\beta \beta}^{\min }\right)_{\mathrm{IO}}=18.4 \pm 1.3 \mathrm{meV},
$$

whose uncertainty is dominated by the uncertainty on the solar mixing angle $\theta_{12}$. Using the latest NuFIT results [27], we obtain $\left(m_{\beta \beta}^{\min }\right)_{\mathrm{IO}}=18.6 \pm 1.2 \mathrm{meV}$. The lower bound on $m_{\beta \beta}$ corresponds to an upper bound on $T_{1 / 2}$ at the scale of $10^{27}-10^{28}$ years, depending on the value of the parameters in Eq. (1). 
TABLE I. Nuclear matrix elements $M$ for $0 v \beta \beta$ decay mediated by light neutrinos, calculated with the NSM, QRPA, EDF, and IBM methods. The ranges correspond to the minimum and maximum values obtained with the same many-body method.

\begin{tabular}{lcccc}
\hline \hline & Ref. & ${ }^{76} \mathrm{Ge}$ & ${ }^{100} \mathrm{Mo}$ & ${ }^{136} \mathrm{Xe}$ \\
\hline NSM & {$[35]$} & $2.89,3.07$ & & $2.28,2.45$ \\
& {$[36]$} & $3.37,3.57$ & & $1.63,1.76$ \\
& {$[37]$} & 2.66 & & 2.39 \\
& All & $2.66-3.57$ & & $1.63-2.45$ \\
QRPA & {$[38]$} & 5.09 & & 1.55 \\
& {$[39]$} & 5.26 & 3.90 & 2.91 \\
& {$[40]$} & 4.85 & 5.87 & 2.72 \\
& {$[41]$} & $3.12,3.40$ & & $1.11,1.18$ \\
& {$[42]$} & & & 3.38 \\
& All & $3.12-5.26$ & $3.90-5.87$ & $1.11-3.38$ \\
EDF & {$[43]$} & 4.60 & 5.08 & 4.20 \\
& {$[44]$} & 5.55 & 6.59 & 4.77 \\
& {$[45]$} & 6.04 & 6.48 & 4.24 \\
& All & $4.60-6.04$ & $5.08-6.59$ & $4.20-4.77$ \\
IBM & {$[46]^{\mathrm{a}}$} & 5.14 & 3.84 & 3.25 \\
& {$[47]$} & 6.34 & 5.08 & 3.40 \\
& All & $5.14-6.34$ & $3.84-5.08$ & $3.25-3.40$ \\
\hline \hline
\end{tabular}

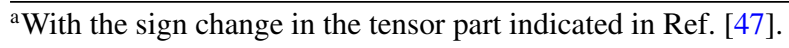

In the last decades, a vast experimental program has been mounted to develop experiments with sensitivity reaching $\left(m_{\beta \beta}^{\min }\right)_{\mathrm{IO}}$, able to exhaustively test whether $0 v \beta \beta$ decay is mediated by the exchange of light neutrinos with invertedordered masses [28,29]. Mature designs are now available for multiple detection techniques, and the physics community is discussing how to proceed. As part of this process, the Astroparticle Physics European Consortium (APPEC) is updating its $0 \nu \beta \beta$ decay road map [29] and the United States Department of Energy has started a ton-scale-experiment portfolio review. Conceptual designs are available for three experiments [30-32], whose construction can start as soon as funding is available. These experiments use different $0 \nu \beta \beta$-decaying isotopes and detection technologies, and can perform independent and complementary measurements.

As mentioned above, observing $0 \nu \beta \beta$ decay would unambiguously demonstrate matter creation and prove the Majorana nature of neutrinos. However, the conversion between $T_{1 / 2}$ and $m_{\beta \beta}$ in Eq. (1) is not trivial and requires inputs from nuclear theory. While the phase-space integral $G$ has been calculated with negligible uncertainty [33], obtaining reliable nuclear matrix elements $M$ is challenging, as it requires computationally intensive many-body calculations and the evaluation of several operators [13,34]. Four primary many-body methods have been historically used in the field: the nuclear shell model (NSM) [35-37], the quasiparticle random-phase approximation (QRPA) method [38-42], energy-density functional (EDF) theory [43-45], and the interacting boson model (IBM) [46,47]. For each of these methods, several calculations have been performed under different assumptions and approximations. The most recent results are listed in Table I. They can differ by up to a fac- tor of three for a given isotope, and significant differences are present even within each method. The spread of values gives a rough idea of the many-body uncertainties on $M$ (additional uncertainty contributions are discussed below). For some methods, calculations are not available for all isotopes.

The reach of $0 \nu \beta \beta$ decay experiments is conventionally expressed in terms of discovery and exclusion sensitivities on $m_{\beta \beta}$. The discovery sensitivity corresponds to the smallest $m_{\beta \beta}$ value for which an experiment has $50 \%$ probability of observing a signal at $99.7 \%$ confidence level (CL). The exclusion sensitivity corresponds to the median $90 \%$-CL upper limit that an experiment will set on $m_{\beta \beta}$ assuming $0 v \beta \beta$ decay is not observable. As stated earlier, fully testing the invertedordering scenario requires sensitivity to $\left(m_{\beta \beta}^{\min }\right)_{\mathrm{IO}}$, accounting for its uncertainty. For discovery mode, this condition is met when the discovery sensitivity reaches the central value of $\left(m_{\beta \beta}^{\min }\right)_{\mathrm{IO}}$ : the $\left(m_{\beta \beta}^{\min }\right)_{\mathrm{IO}}$ uncertainty is symmetric, so the probability of lower or upper fluctuations is the same, and the $50 \%$ probability for an observation is preserved. However, for exclusion mode, the $\left(m_{\beta \beta}^{\min }\right)_{\mathrm{IO}}$ uncertainty reduces the CL by a variable amount that depends on the experimental parameters, mainly the background statistical uncertainty. Therefore the exclusion sensitivity on $m_{\beta \beta}$ cannot be used to set an experiment-independent condition corresponding to fully covering the inverted-ordering scenario. The discovery sensitivity is anyway the most appropriate metric for searches aiming to discover a process. Thus, reaching a discovery sensitivity of $18.4 \mathrm{meV}$ is the right concrete goal for experiments aiming to explore the full inverted-ordering parameter space.

Figure 1 shows the discovery and exclusion sensitivities of proposed future experiments. We converted the $T_{1 / 2}$ sensitivity values quoted by the LEGEND [31], CUPID [30], and nEXO [48] collaborations to $m_{\beta \beta}$ values using the nuclear matrix elements of Table I. We group the calculations by many-body method to aid in the comparison of more consistent quantities. Remarkably, these experiments all show sensitivity to measure a $0 v \beta \beta$ decay signal at the bottom of the invertedordering parameter space. Some many-body methods, such as EDF theory and IBM, give systematically larger $M$ values, pushing the $m_{\beta \beta}$ sensitivity even lower. QRPA calculations on the other hand give a broad range of results, partially due to the role of nuclear deformation in this framework. The NSM provides $M$ values which are typically smaller than for the other methods, but are not available for ${ }^{100} \mathrm{Mo}$.

Comparing the performance of experiments using different isotopes is challenging because of the large uncertainties affecting the nuclear matrix element predictions. In particular, each many-body method uses different approximations, which are likely to result in a common over- or underestimation of the calculated $M$ values. Even comparisons considering one many-body method at a time can raise concerns. The number of calculations available for each isotope and method can be significantly different, suggesting that not all many-body approaches are equally suitable for all isotopes. Consequently the range of $M$ values cannot be quantitatively interpreted as the uncertainty, which currently remains unknown. One might be tempted to make comparisons based on the central value of the $m_{\beta \beta}$ sensitivity within a specific nuclear method, but this value can be disproportionately affected by a single outlier 

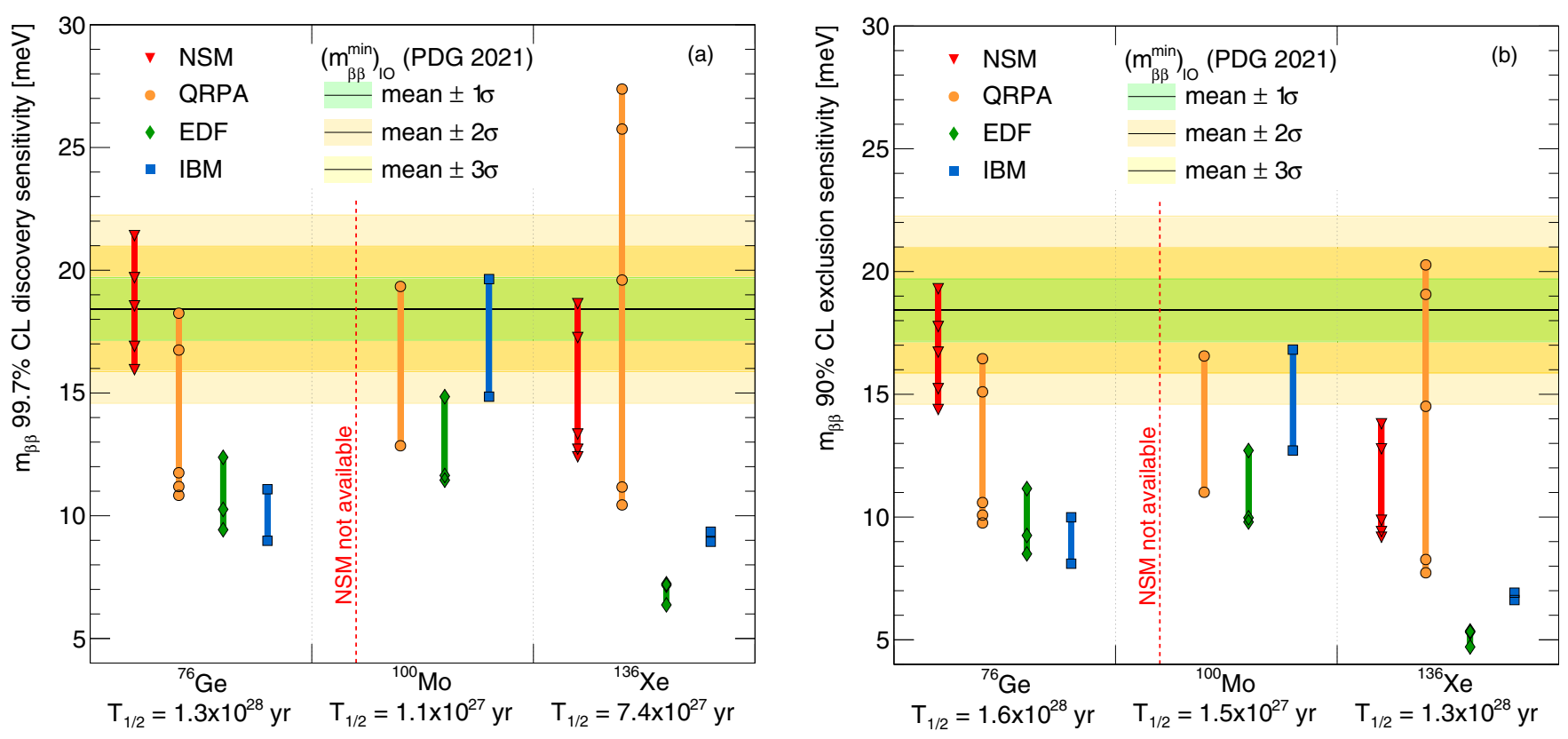

FIG. 1. Comparison of $m_{\beta \beta} 99.7 \%$-CL discovery and 90\%-CL median exclusion sensitivities for different isotopes at stated half-life sensitivities [30,31,48], grouped by nuclear many-body frameworks with matrix element ranges from Table I. The horizontal bands show the variation on $\left(m_{\beta \beta}^{\mathrm{min}}\right)_{\mathrm{IO}}$ under variation of the neutrino oscillation parameters.

matrix element. Weighted averages are also problematic, as the weight given to each calculation would be to some extent arbitrary. Given the lack of objective criteria to compare experimental sensitivities in different isotopes, and the lack of a clear estimate of the uncertainties, we advocate to refrain from ranking experiments' reach quantitatively, and focus instead on the fact that we have a global, multi-isotope endeavor that will fully test the inverted-ordering scenario.

A broad effort to reduce uncertainties is ongoing within the nuclear theory community, with significant advances made in the last few years. Ab initio calculations that incorporate wider nuclear correlations and two-body currents have recently succeeded in predicting single- $\beta$ decay rates [49] with no need for "quenching" - an ad hoc reduction of the value of calculated matrix elements involving the nuclear spin required by less sophisticated calculations [13]. The first available ab initio $0 \nu \beta \beta$ matrix element calculations in medium-sized nuclei, supported by studies in lighter systems [50,51], indicate a relatively mild suppression by tens of percent with respect to the lower limit of the ranges given in Table I [52]. Efforts are underway to improve the quality of these results, extend them to heavier nuclei, and include two-body currents at finite momentum transfers [53]. On the other hand, the contact term introduced in Refs. [34,54], which until recently went unrecognized, is a leading-order contribution to $M$. Effective field theory and $a b$ initio nuclear structure provide a scheme for estimating this contribution [55,56]. A first study in ${ }^{48} \mathrm{Ca}$ suggests that this term can enhance $M$ by about $40 \%$ percent [57], leading to a faster decay rate. In heavier systems, preliminary results suggest a roughly similar impact for all $0 \nu \beta \beta$ isotopes, only slightly dependent on the nuclear many-body method [58]. Complementary studies using, e.g., lattice QCD $[59,60]$ will test whether this claimed enhancement is robust. If so, it may compensate the reduction in decay rate due to the inclusion of the "quenching" physics, leading to a picture similar to the one represented by Fig. 1. Thus, should the current theoretical results be confirmed, the proposed global $0 v \beta \beta$ decay experimental effort would still fully probe the inverted-ordering parameter space.

In this Letter, we have focused on the inverted-ordering scenario as a prominent goalpost for the proposed experimental $0 v \beta \beta$ decay program. However, the discovery power of these experiments is high even assuming other, equally reasonable scenarios. By reaching a sensitivity of the order of tens of $\mathrm{meV}$, these searches will probe a significant fraction of the remaining parameter space for left-handed neutrino exchange even if neutrino masses follow the normal ordering. Bayesian analyses suggest up to $50 \%$ discovery probabilities for the normal ordering scenario [61,62], and a nonvanishing discovery probability even assuming the most unfavorable value of the Majorana phases [63]. Significant advancement would also be made in probing the exchange of heavy mediators. For many such models, $0 v \beta \beta$ decay searches probe energy scales beyond the reach of current accelerator technology [9]. Additional physics mechanisms could completely change the parameter space of interest, potentially even increasing the discovery power of future experiments [64-67]. In general, pushing $0 \nu \beta \beta$ decay sensitivity to increasingly large half-life values explores uncharted territory, and new physics could manifest at any time.

We would like to thank Christoph Wiesinger and Steven R. Elliott for valuable discussions. This work has been supported by the Science and Technology Facilities Council, part of U.K. Research and Innovation (Grant No. ST/T004169/1), 
the EU Horizon 2020 research and innovation program under the Marie Skłodowska-Curie Grant Agreement No. 754496, the Spanish MICINN through the "Ramón y Cajal" program with Grant No. RYC-2017-22781, the Italian Research Grant No. 2017W4HA7S "NAT-NET: Neutrino and
Astroparticle Theory Network" under the program PRIN 2017 funded by MIUR, the AEI "Unit of Excellence María de Maeztu 2020-2023" Award No. CEX2019-000918-M, the AEI Grant No. FIS2017-87534-P, and the U.S. DOE Office of Nuclear Physics under Grant No. DE-FG02-97ER41020.
[1] T. Kajita, Nobel Lecture: Discovery of atmospheric neutrino oscillations, Rev. Mod. Phys. 88, 030501 (2016).

[2] A. B. McDonald, Nobel lecture: The sudbury neutrino observatory: Observation of flavor change for solar neutrinos, Rev. Mod. Phys. 88, 030502 (2016).

[3] K. Eguchi et al. (KamLAND), First Results from KamLAND: Evidence for Reactor Anti-Neutrino Disappearance, Phys. Rev. Lett. 90, 021802 (2003).

[4] P. A. Zyla et al. (Particle Data Group), Review of particle physics, Prog. Theor. Exp. Phys. 2020, 083C01 (2020), and 2021 update.

[5] W. H. Furry, On transition probabilities in double betadisintegration, Phys. Rev. 56, 1184 (1939).

[6] J. Schechter and J. W. F. Valle, Neutrinoless double- $\beta$ decay in SU(2) $\times U(1)$ theories, Phys. Rev. D 25, 2951 (1982).

[7] M. Fukugita and T. Yanagida, Baryogenesis without grand unification, Phys. Lett. B 174, 45 (1986).

[8] F. F. Deppisch, P. S. Bhupal Dev, and A. Pilaftsis, Neutrinos and collider physics, New J. Phys. 17, 075019 (2015).

[9] T. Peng, M. J. Ramsey-Musolf, and P. Winslow, TeV lepton number violation: From neutrinoless double- $\beta$ decay to the LHC, Phys. Rev. D 93, 093002 (2016).

[10] J. D. Vergados, H. Ejiri, and F. Simkovic, Theory of neutrinoless double beta decay, Rep. Prog. Phys. 75, 106301 (2012).

[11] A. de Gouvea and J. Jenkins, A survey of lepton number violation via effective operators, Phys. Rev. D 77, 013008 (2008).

[12] M. Mitra, G. Senjanovic, and F. Vissani, Neutrinoless double beta decay and heavy sterile neutrinos, Nucl. Phys. B 856, 26 (2012).

[13] J. Engel and J. Menéndez, Status and future of nuclear matrix elements for neutrinoless double-beta decay: A review, Rep. Prog. Phys. 80, 046301 (2017).

[14] B. Märkisch et al., Measurement of the Weak Axial-Vector Coupling Constant in the Decay of Free Neutrons Using a Pulsed Cold Neutron Beam, Phys. Rev. Lett. 122, 242501 (2019).

[15] S. M. Bilenky and C. Giunti, Neutrinoless double-beta decay: A probe of physics beyond the standard model, Int. J. Mod. Phys. A 30, 1530001 (2015).

[16] F. Vissani, Signal of neutrinoless double beta decay, neutrino spectrum and oscillation scenarios, J. High Energy Phys. 06 (1999) 022.

[17] K. Abe et al. (Super-Kamiokande), Solar neutrino measurements in Super-Kamiokande IV, Phys. Rev. D 94, 052010 (2016).

[18] K. Abe et al. (Super-Kamiokande), Atmospheric neutrino oscillation analysis with external constraints in Super-Kamiokande I-IV, Phys. Rev. D 97, 072001 (2018).

[19] K. Abe et al. (T2K), Constraint on the matter-antimatter symmetry-violating phase in neutrino oscillations, Nature (London) 580, 339 (2020); Publisher Correction: Constraint on the matterantimatter symmetry-violating phase in neutrino oscillations, 583, E16(E) (2020).

[20] M. A. Acero et al. (NOvA), First Measurement of Neutrino Oscillation Parameters using Neutrinos and Antineutrinos by NOvA, Phys. Rev. Lett. 123, 151803 (2019).

[21] P. Adamson et al. (MINOS+), Precision Constraints for ThreeFlavor Neutrino Oscillations from the Full MINOS+ and MINOS Dataset, Phys. Rev. Lett. 125, 131802 (2020).

[22] M. G. Aartsen et al. (IceCube), Measurement of Atmospheric Neutrino Oscillations at 6-56 GeV with IceCube DeepCore, Phys. Rev. Lett. 120, 071801 (2018)

[23] D. Adey et al. (Daya Bay), Measurement of the Electron Antineutrino Oscillation with 1958 Days of Operation at Daya Bay, Phys. Rev. Lett. 121, 241805 (2018).

[24] G. Bak et al. (RENO), Measurement of Reactor Antineutrino Oscillation Amplitude and Frequency at RENO, Phys. Rev. Lett. 121, 201801 (2018).

[25] H. de Kerret et al. (Double Chooz), Double Chooz $\theta_{13}$ measurement via total neutron capture detection, Nat. Phys. 16, 558 (2020).

[26] A. Gando et al. (KamLAND), Reactor on-off antineutrino measurement with KamLAND, Phys. Rev. D 88, 033001 (2013).

[27] I. Esteban, M. C. Gonzalez-Garcia, M. Maltoni, T. Schwetz, and A. Zhou, The fate of hints: Updated global analysis of threeflavor neutrino oscillations, J. High Energy Phys. 09 (2020) 178.

[28] NSAC NLDBD Subcommittee, Report to the Nuclear Science Advisory Committee: Neutrinoless double beta decay, http://science.energy.gov/np/nsac/reports.

[29] A. Giuliani, J. J. Gomez Cadenas, S. Pascoli, E. Previtali, R. Saakyan, K. Schäffner, and S. Schönert (APPEC Committee), Double beta decay APPEC committee report, https://inspirehep. net/literature/1758439.

[30] W. R. Armstrong et al. (CUPID), CUPID pre-CDR, arXiv:1907.09376.

[31] N. Abgrall et al. (LEGEND), LEGEND-1000 preconceptual design report, arXiv:2107.11462.

[32] S. A. Kharusi et al. (nEXO), nEXO preconceptual design report, arXiv: 1805.11142 .

[33] J. Kotila and F. Iachello, Phase space factors for double- $\beta$ decay, Phys. Rev. C 85, 034316 (2012).

[34] V. Cirigliano, W. Dekens, E. Mereghetti, and A. Walker-Loud, Neutrinoless double- $\beta$ decay in effective field theory: The light-Majorana neutrino-exchange mechanism, Phys. Rev. C 97, 065501 (2018); Erratum: Neutrinoless double- $\beta$ decay in effective field theory: The light-Majorana neutrino-exchange mechanism [Phys. Rev. C 97, 065501 (2018)], 100, 019903(E) (2019).

[35] J. Menéndez, Neutrinoless $\beta \beta$ decay mediated by the exchange of light and heavy neutrinos: The role of nuclear structure correlations, J. Phys. G 45, 014003 (2018).

[36] M. Horoi and A. Neacsu, Shell model predictions for ${ }^{124} \mathrm{Sn}$ double- $\beta$ decay, Phys. Rev. C 93, 024308 (2016). 
[37] L. Coraggio, A. Gargano, N. Itaco, R. Mancino, and F. Nowacki, The calculation of the neutrinoless double-beta decay matrix element within the realistic shell model, Phys. Rev. C 101, 044315 (2020).

[38] M. T. Mustonen and J. Engel, Large-scale calculations of the double- $\beta$ decay of ${ }^{76} \mathrm{Ge},{ }^{130} \mathrm{Te},{ }^{136} \mathrm{Xe}$, and ${ }^{150} \mathrm{Nd}$ in the deformed self-consistent Skyrme quasiparticle random-phase approximation, Phys. Rev. C 87, 064302 (2013).

[39] J. Hyvarinen and J. Suhonen, Nuclear matrix elements for $0 \nu \beta \beta$ decays with light or heavy Majorana-neutrino exchange, Phys. Rev. C 91, 024613 (2015).

[40] F. Šimkovic, A. Smetana, and P. Vogel, $0 v \beta \beta$ nuclear matrix elements, neutrino potentials, and SU(4) symmetry, Phys. Rev. C 98, 064325 (2018).

[41] D.-L. Fang, A. Faessler, and F. Šimkovic, $0 v \beta \beta$-decay nuclear matrix element for light and heavy neutrino mass mechanisms from deformed quasiparticle random-phase approximation calculations for ${ }^{76} \mathrm{Ge},{ }^{82} \mathrm{Se},{ }^{130} \mathrm{Te},{ }^{136} \mathrm{Xe}$, and ${ }^{150} \mathrm{Nd}$ with isospin restoration, Phys. Rev. C 97, 045503 (2018).

[42] J. Terasaki, Strength of the isoscalar pairing interaction determined by a relation between double-charge change and double-pair transfer for double- $\beta$ decay, Phys. Rev. C 102, 044303 (2020).

[43] T. R. Rodriguez and G. Martinez-Pinedo, Energy Density Functional Study of Nuclear Matrix Elements for Neutrinoless $\beta \beta$ Decay, Phys. Rev. Lett. 105, 252503 (2010).

[44] N. López Vaquero, T. R. Rodríguez, and J. L. Egido, Shape and Pairing Fluctuation Effects on Neutrinoless Double-Beta Decay Nuclear Matrix Elements, Phys. Rev. Lett. 111, 142501 (2013).

[45] L. S. Song, J. M. Yao, P. Ring, and J. Meng, Nuclear matrix element of neutrinoless double- $\beta$ decay: Relativity and short-range correlations, Phys. Rev. C 95, 024305 (2017).

[46] J. Barea, J. Kotila, and F. Iachello, $0 v \beta \beta$ and $2 v \beta \beta$ nuclear matrix elements in the interacting boson model with isospin restoration, Phys. Rev. C 91, 034304 (2015).

[47] F. F. Deppisch, L. Graf, F. Iachello, and J. Kotila, Analysis of light neutrino exchange and short-range mechanisms in $0 v \beta \beta$ decay, Phys. Rev. D 102, 095016 (2020).

[48] G. Adhikari et al. (nEXO), nEXO: Neutrinoless double beta decay search beyond $10^{28}$ year half-life sensitivity, arXiv:2106.16243.

[49] P. Gysbers et al., Discrepancy between experimental and theoretical $\beta$-decay rates resolved from first principles, Nat. Phys. 15, 428 (2019).

[50] J. M. Yao, B. Bally, J. Engel, R. Wirth, T. R. Rodríguez, and H. Hergert, $A b$ Initio Treatment of Collective Correlations and the Neutrinoless Double Beta Decay of ${ }^{48} \mathrm{Ca}$, Phys. Rev. Lett. 124, 232501 (2020).

[51] S. Novario, P. Gysbers, J. Engel, G. Hagen, G. R. Jansen, T. D. Morris, P. Navrátil, T. Papenbrock, and S. Quaglioni, Coupled-
Cluster Calculations of Neutrinoless Double- $\beta$ Decay in ${ }^{48} \mathrm{Ca}$, Phys. Rev. Lett. 126, 182502 (2021).

[52] A. Belley, C. G. Payne, S. R. Stroberg, T. Miyagi, and J. D. Holt, Ab Initio Neutrinoless Double-Beta Decay Matrix Elements for ${ }^{48} \mathrm{Ca},{ }^{76} \mathrm{Ge}$, and ${ }^{82} \mathrm{Se}$, Phys. Rev. Lett. 126, 042502 (2021).

[53] J. Menéndez, D. Gazit, and A. Schwenk, Chiral Two-Body Currents in Nuclei: Gamow-Teller Transitions and Neutrinoless Double-Beta Decay, Phys. Rev. Lett. 107, 062501 (2011).

[54] V. Cirigliano, W. Dekens, J. de Vries, M. L. Graesser, E. Mereghetti, S. Pastore, and U. van Kolck, New Leading Contribution to Neutrinoless Double- $\beta$ Decay, Phys. Rev. Lett. 120, 202001 (2018).

[55] V. Cirigliano, W. Dekens, J. de Vries, M. Hoferichter, and E. Mereghetti, Toward Complete Leading-Order Predictions for Neutrinoless Double $\beta$ Decay, Phys. Rev. Lett. 126, 172002 (2021).

[56] V. Cirigliano, W. Dekens, J. de Vries, M. Hoferichter, and E. Mereghetti, Determining the leading-order contact term in neutrinoless double $\beta$ decay, J. High Energy Phys. 05 (2021) 289.

[57] R. Wirth, J. M. Yao, and H. Hergert, Ab initio calculation of the contact operator contribution in the standard mechanism for neutrinoless double beta decay, arXiv:2105.05415.

[58] L. Jokiniemi, P. Soriano, and J. Menéndez, Impact of the leading-order short-range nuclear matrix element on the neutrinoless double-beta decay of heavy nuclei, arXiv:2107.13354.

[59] V. Cirigliano, W. Detmold, A. Nicholson, and P. Shanahan, Lattice QCD inputs for nuclear double beta decay, arXiv:2003.08493.

[60] Z. Davoudi and S. V. Kadam, Path from Lattice QCD to the Short-Distance Contribution to $0 v \beta \beta$ Decay with a Light Majorana Neutrino, Phys. Rev. Lett. 126, 152003 (2021).

[61] M. Agostini, G. Benato, and J. A. Detwiler, Discovery probability of next-generation neutrinoless double- $\beta$ decay experiments, Phys. Rev. D 96, 053001 (2017).

[62] A. Caldwell, A. Merle, O. Schulz, and M. Totzauer, Global Bayesian analysis of neutrino mass data, Phys. Rev. D 96, 073001 (2017).

[63] M. Agostini, G. Benato, S. Dell'Oro, S. Pirro, and F. Vissani, Discovery probabilities of Majorana neutrinos based on cosmological data, Phys. Rev. D 103, 033008 (2021).

[64] V. Cirigliano, W. Dekens, J. de Vries, M. L. Graesser, and E. Mereghetti, A neutrinoless double beta decay master formula from effective field theory, J. High Energy Phys. 12 (2018) 097.

[65] V. Tello, M. Nemevsek, F. Nesti, G. Senjanovic, and F. Vissani, Left-Right Symmetry: From LHC to Neutrinoless Double Beta Decay, Phys. Rev. Lett. 106, 151801 (2011).

[66] S. F. King, A. Merle, and A. J. Stuart, The power of neutrino mass sum rules for neutrinoless double beta decay experiments, J. High Energy Phys. 12 (2013) 005.

[67] W. Rodejohann, Neutrino-less double beta decay and particle physics, Int. J. Mod. Phys. E 20, 1833 (2011). 